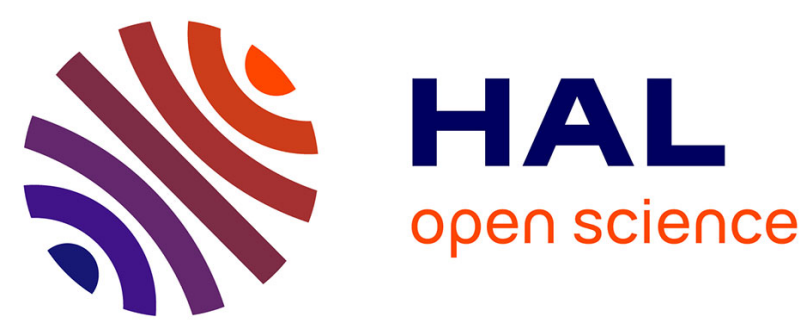

\title{
Etude de la conduction électrique dans le semiconducteur chalcogénure As35Te28Ge16S21 en liaison avec la constitution de charges d'espace
}

\author{
M. C. Félix, J. Fornazéro, J.M. Mackovski, R. Ongaro
}

\section{- To cite this version:}

M. C. Félix, J. Fornazéro, J.M. Mackovski, R. Ongaro. Etude de la conduction électrique dans le semiconducteur chalcogénure As35Te28Ge16S21 en liaison avec la constitution de charges d'espace. Revue de Physique Appliquée, 1980, 15 (1), pp.37-43. 10.1051/rphysap:0198000150103700 . jpa00244696

\section{HAL Id: jpa-00244696 https://hal.science/jpa-00244696}

Submitted on 1 Jan 1980

HAL is a multi-disciplinary open access archive for the deposit and dissemination of scientific research documents, whether they are published or not. The documents may come from teaching and research institutions in France or abroad, or from public or private research centers.
L'archive ouverte pluridisciplinaire HAL, est destinée au dépôt et à la diffusion de documents scientifiques de niveau recherche, publiés ou non, émanant des établissements d'enseignement et de recherche français ou étrangers, des laboratoires publics ou privés. 


\title{
Etude de la conduction électrique dans le semiconducteur chalcogénure $\mathrm{As}_{35} \mathbf{T e}_{28} \mathrm{Ge}_{16} \mathrm{~S}_{21}$ en liaison avec la constitution de charges d'espace
}

\author{
M. C. Félix, J. Fornazéro, J. M. Mackovski, R. Ongaro \\ Université Claude-Bernard, Lyon-I, Laboratoire d'Electricité \\ 43, boulevard du 11-Novembre-1918, Bâtiment 721, 69621 Villeurbanne, France \\ (Reçu le 16 juillet 1979, révisé le 28 septembre 1979, accepté le 2 octobre 1979)
}

\begin{abstract}
Résumé. - L'étude des propriétés électriques d'échantillons épais de $\mathrm{As}_{35} \mathrm{Te}_{28} \mathrm{Ge}_{16} \mathrm{~S}_{21}$ a permis de mettre en évidence des caractéristiques inhabituelles pour les chalcogénures ; en particulier un effet de mémoire apparaît sur la conductivité électronique mesurée à basse température. Nous proposons un modèle basé sur l'existence de couches superficielles plus résistantes, entraînant la formation de charges d'espace qui seraient à l'origine des phénomènes observés.
\end{abstract}

\begin{abstract}
The study of electrical properties of thick samples of $\mathrm{As}_{35} \mathrm{Te}_{28} \mathrm{Ge}_{16} \mathrm{~S}_{21}$ gives evidence for some unusual characteristics in chalcogenide materials ; in particular, a memory-effect has been revealed in the electronic conductivity measured in the low temperature range. We propose a model in which two superficial layers more resistive than the bulk, cause a space charge buildup, which would give rise to the observed phenomena.
\end{abstract}

1. Introduction. - Dans les verres chalcogénures, Street et Mott [1] considèrent qu'il existe des densités élevées $\left(>10^{18} \mathrm{~cm}^{-3}\right)$ de défauts ponctuels associés à des liaisons pendantes (dangling bonds), ce qui se traduit par la présence d'états localisés nombreux dans la bande interdite. Utilisant l'hypothèse d'Anderson [2] selon laquelle toute variation du nombre d'électrons occupant une liaison pendante entraîne une forte distorsion du réseau, Street et Mott concluent qu'il n'est plus possible d'affecter une seule énergie à chaque état localisé. De ce fait, les liaisons pendantes peuvent constituer des centres de recombinaison $\mathrm{D}^{0}$ ou bien des pièges $\mathrm{D}^{+}$et $\mathrm{D}^{-}$susceptibles de jouer respectivement le rôle de donneurs ou d'accepteurs. Adler et Yoffa [3] ont montré que, dans ces conditions, le niveau de Fermi se trouve fixé à peu près au milieu de la bande interdite et que la conductivité à température ambiante a tendance à être de type $\mathrm{p}$.

Pour les températures moyennes et élevées, on observe généralement une conduction activée, l'énergie d'activation étant de l'ordre de la moitié du gap optique. Cette conductivité [4], [5] dépend de la tension appliquée suivant la loi :

$$
\sigma=\sigma_{0} \exp \left(-\frac{\Delta E}{k T}\right) \exp \left(\frac{V}{V_{0}}\right)^{n}
$$

Le paramètre $V_{0}$ étant une fonction croissante de la température, la valeur absolue de la pente des droites obtenues dans le diagramme $(\log i, 1 / T)$, est une fonction décroissante de $V$ [6]. Par ailleurs, l'exposant $n$ prend la valeur $1 / 2$ lorsque la conduction fait intervenir un mécanisme de Poole-Frenkel d'ionisation de pièges profonds sous l'influence du champ électrique ; la valeur 1 est caractéristique des chalcogénures amorphes et peut correspondre, selon Fagen et Fritzsche [5], à un recouvrement partiel des puits de potentiel.

Dans le domaine des basses températures apparaissent très souvent des courbes à faibles pentes de la forme :

$$
\sigma=\sigma_{0} \exp \left(-\left(\frac{T_{0}}{T}\right)^{1 / 4}\right)
$$

Mott [7] les attribue à une conduction par sauts, assisté par phonons, au voisinage du niveau de Fermi. Ces paliers étant parfois obtenus et modifiés par des recuits, ils sont aussi attribués à une dévitrification et à une recristallisation partielle [5]. On remarquera que, de ces deux interprétations, l'une repose sur l'application d'un modèle de bande à un solide homogène, l'autre suppose le solide essentịellement hétérogène. Il semble cependant qu'on 
puisse concilier ces deux points de vue en utilisant la théorie des percolations et retrouver, comme le font Pike et Seager, la loi en $T^{-1 / 4}[8]$.

En ce qui concerne les charges d'espace qui s'établissent dans les amorphes, peu d'études quantitatives ont été publiées. Par contre, leur présence, au voisinage des électrodes en particulier, est souvent invoquée pour rendre compte, par exemple, du mécanisme d'initiation électronique de la commutation [9], [10].

La présente étude concerne les propriétés dans l'état peu conducteur (OFF) d'échantillons épais du chalcogénure $\mathrm{As}_{35} \mathrm{Te}_{28} \mathrm{Ge}_{16} \mathrm{~S}_{21}$, dont la composition a été choisie pour obtenir une bonne stabilité thermique de l'état amorphe, associée à des conductivités électriques comprises entre $10^{-7}$ et $10^{-10} \Omega^{-1} \mathrm{~cm}^{-1}$ à l'ambiante dans l'état OFF. Nous analyserons les variations de la conductivité en fonction de la température et de la tension appliquée, les courants thermostimulés et de thermodépolarisation et les distributions de potentiel. Nous mettrons ainsi en évidence l'influence très nette des charges d'espace constituées sous l'action des champs électriques appliqués ; ce qui nous permettra d'interpréter certains comportements expérimentaux inhabituels dans les chalcogénures, en particulier la mémoire que les paliers conservent des précédents traitements électriques bien que les tensions appliquées soient très inférieures à la tension de seuil.

\section{Préparation des échantillons et dispositif expéri-} mental. - Des carottes de $\mathrm{As}_{35} \mathrm{Te}_{28} \mathrm{Ge}_{16} \mathrm{~S}_{21}$ ont été préparées par fusion du mélange puis solidification de l'alliage sous vide [11].

Des plaquettes d'épaisseurs comprises entre $0,7 \mathrm{~mm}$ et $1 \mathrm{~mm}$ ont été obtenues par sciage. Leurs faces sont polies mécaniquement dans un courant d'eau. Les échantillons sont alors nettoyés par ultra-sons dans de l'eau distillée puis de l'alcool pur. Dans cette étude, les électrodes sont constituées par des dépôts de vernis conducteur à l'argent.

Une cellule de mesure, soigneusement blindée, permet de mesurer des courants jusqu'à $10^{-14} \mathrm{~A}$ avec un électromètre Keithley $610 \mathrm{C}$. La gamme des températures utilisées varie de $130 \mathrm{~K}$ à $300 \mathrm{~K}$ et il est possible d'obtenir des remontées linéaires de température.

Une cellule et un appareillage indépendants [12] permettent de déterminer les distributions de potentiel à la température ambiante.

Entre deux applications de la tension les échantillons sont toujours maintenus en court-circuit.

3. Résultats expérimentaux. - 3.1 CouRANTS DE CONDUCTION. - L'étude des propriétés électriques du composé $A_{35} \mathrm{Te}_{28} \mathrm{Ge}_{16} \mathrm{~S}_{21}$ a été abordée par la mesure du courant de conduction $i_{\mathrm{c}}$ dans l'intervalle de températures $130 \mathrm{~K}-300 \mathrm{~K}$ et pour des tensions $V$ comprises entre $0,1 \mathrm{~V}$ et $360 \mathrm{~V}$.
Des mesures de pouvoir thermoélectrique ont montré que la conductivité à l'ambiante est due à des porteurs de type $p$ [13].

3.1.1 Influence de la température. - Dans ces expériences, la tension est appliquée à la température ambiante pendant un temps suffisant pour que le courant soit stabilisé. On fait ensuite décroître très lentement la température jusqu'à $130 \mathrm{~K}$ tout en mesurant le courant $i_{\mathrm{c}}(T)$. Pour chaque échantillon, on construit un réseau de courbes avec $V$ pour paramètre. L'ensemble des mesures réalisées permet de définir deux types de comportements :

- Pour le premier (courbes 1 et $1^{\prime}$ de la figure 1), la conductivité est activée dans toute la gamme de températures explorée. L'énergie d'activation $E_{\mathrm{a}}$, comprise entre 0,55 et $0,60 \mathrm{eV}$, est légèrement inférieure à la moitié du gap optique dont la valeur est, selon Fagen et Fritzsche [14], de l'ordre de 1,2 eV.

- Pour le second (courbes 2 et $2^{\prime}$ de la figure 1), la conductivité reste activée jusque vers $200 \mathrm{~K}$ puis apparaît un palier, $i_{\mathrm{c}}$ décroissant faiblement ; la zone de transition débute à une température qui dépend de l'importance relative des valeurs de $i_{\mathrm{c}}$, à basse température et à la température ambiante. Ces paliers admettent une représentation linéaire dans le diagramme $\left(\log i, T^{-1 / 4}\right)$ mais ceci ne suffit pas à légitimer l'application de la loi de Mott [7]. En effet, les valeurs trouvées pour le paramètre $T_{0}$ $\left(<6 \times 10^{4} \mathrm{~K}\right)$ ont une reproductibilité insuffisante et un ordre de grandeur notablement inférieur à la valeur attendue $\left(\simeq 10^{6} \mathrm{~K}\right)$. Nous avons vérifié que

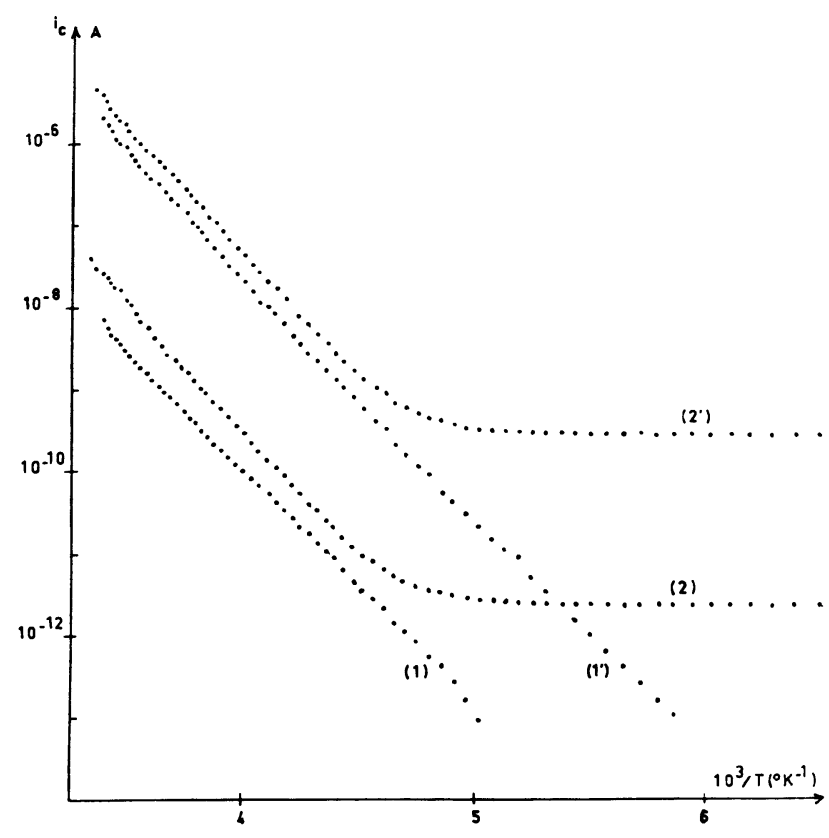

Fig. 1. - Courants de conduction en fonction de la température pour différentes tensions appliquées. (1) et (2) sous $1 \mathrm{~V} ;\left(1^{\prime}\right)$ sous $50 \mathrm{~V} ;\left(2^{\prime}\right)$ sous $100 \mathrm{~V}$.

[Conduction currents versus temperature for different applied voltages. (1) and (2) : $\left.1 \mathrm{~V} ;\left(1^{\prime}\right): 50 \mathrm{~V} ;\left(2^{\prime}\right): 100 \mathrm{~V}.\right]$ 
le courant $i_{\mathrm{c}}(T)$ peut être considéré comme la somme de deux composantes indépendantes. Ceci nous amène à considérer la résistance de l'échantillon comme résultant de la mise en parallèle de deux résistances $R_{0} \exp \left(+E_{\mathrm{a}} / k T\right)$ et $R_{1}$.

3.1.2 Influence de la tension appliquée. - Dans le but d'élucider les mécanismes de la conduction, nous nous sommes proposés, dans cette deuxième partie, d'étudier l'influence de la tension appliquée sur les différentes caractéristiques de la conduction.

- Courants de conduction à la température ambiante $T_{0}$ : L'échantillon est soumis à des tensions successives de valeurs croissantes, chacune d'entre elles étant maintenue pendant un laps de temps suffisant pour que le courant permanent $i_{\mathrm{c}}\left(T_{0}\right)$ soit atteint. Jusqu'à $340 \mathrm{~V}, i_{\mathrm{c}}$ est proportionnel à $V$ (courbe 1 de la figure 2). Au-delà de $340 \mathrm{~V}, i_{\mathrm{c}}$ croît de plus en plus vite avec $V$, lorsqu'on approche de la tension de seuil correspondant au passage à l'état $O N$.

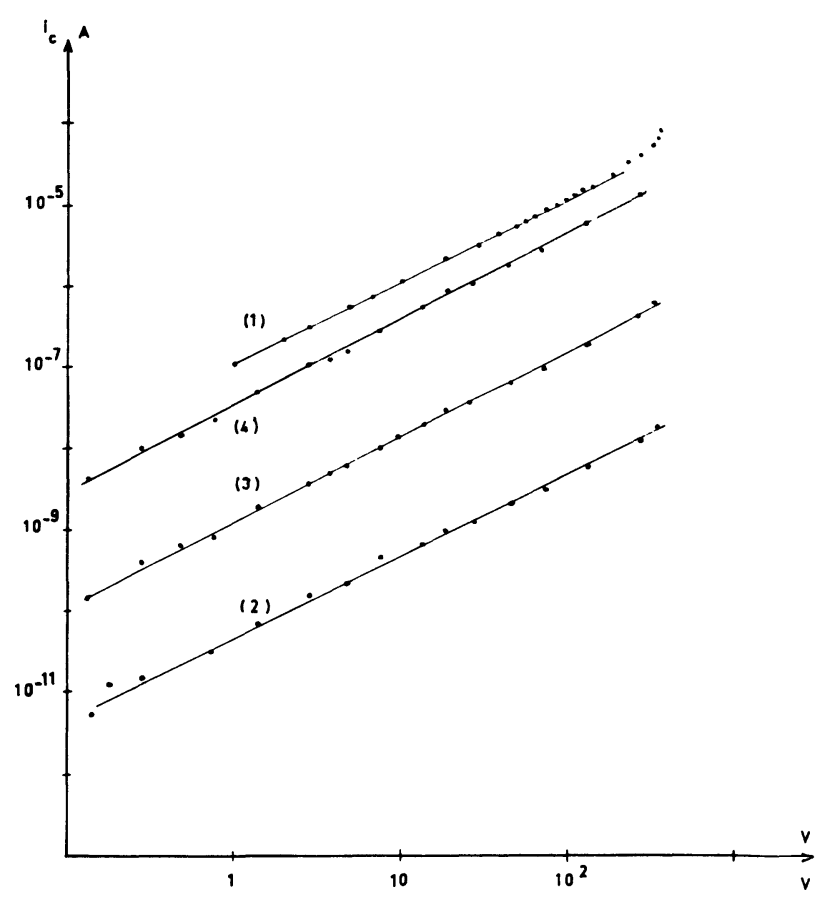

Fig. 2. - Courants de conduction en fonction de la tension appliquée. (1) : température ambiante ; (2) : $222 \mathrm{~K}$; (3) : $250 \mathrm{~K}$; (4) : $280 \mathrm{~K}$.

[Conduction currents versus applied voltage. (1) : room temperature ; (2) : $222 \mathrm{~K}$; (3) : $250 \mathrm{~K}$; (4) : $280 \mathrm{~K}$.]

- Conductivité dans la région de conduction activée : A partir d'un réseau de courbes $i_{\mathrm{c}}(T)$ tracées pour différentes tensions (étude du paragraphe 1.1.1), on vérifie que, pour toute température $T_{\mathrm{n}}$ de la région activée, $i_{\mathrm{c}}\left(T_{\mathrm{n}}\right) \propto V^{\alpha}$ avec $\alpha \# 1$. Par exemple, pour les températures $222 \mathrm{~K}, 250 \mathrm{~K}$ et $285 \mathrm{~K}$, $\alpha$ prend les valeurs 0,99-1,04-1,05 respectivement (courbes 2, 3 et 4 de la figure 2). La composante activée du courant a donc un comportement ohmique.
- Energie d'activation : L'énergie d'activation $E_{\mathrm{a}}$, déduite de la pente des droites obtenues dans le diagramme $\left(\log i_{\mathrm{c}}, 1 / T\right)$ de la figure 1 , est une fonction croissante de $V$ (Fig. 3). Ainsi le sens de variation de $E_{\mathrm{a}}$ vis-à-vis de $V$ est opposé à celui que permet de prévoir la relation (1). On peut remarquer cependant que le champ électrique maximum utilisé ici $\left(<6 \times 10^{3} \mathrm{~V} \cdot \mathrm{cm}^{-1}\right)$ est de l'ordre du centième des champs appliqués aux couches minces. Dans cette gamme et si $V_{0}$ est assez grand, la relation (1) donnerait une diminution faible.

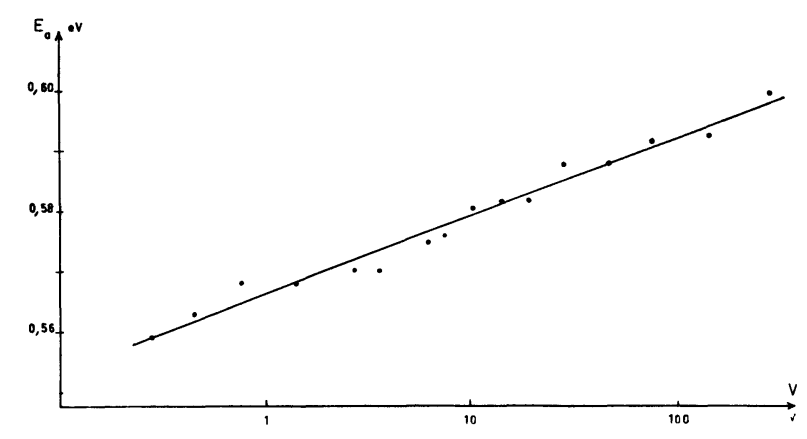

Fig. 3. - Energie d'activation en fonction de la tension appliquée. [Activation energy versus applied voltage.]

- Hauteur des paliers basse température : Lorsqu'on relève le courant de conduction $i_{\mathrm{cl}}$ à $130 \mathrm{~K}$, obtenu dans une séquence de tensions croissantes au cours des mesures $i_{\mathrm{c}}(T)$, on observe une augmentation régulière avec $V$ (par exemple séquence 3-45-6 de la figure 4 où $\left.i_{\mathrm{c}} \propto V^{2}\right)$. Si cependant après avoir appliqué une tension élevée, on revient à une valeur faible de $V$, la valeur de $i_{\text {cl }}$ obtenue alors est supérieure à celle précédemment trouvée pour une tension comparable (point 7). Par ailleurs, une inversion du signe de la tension $(7 \rightarrow 8)$ provoque une diminution du module de $i_{\mathrm{cl}}$ par rapport à sa valeur dans la séquence de signe opposé. Tout se passe comme si l'échantillon était doté d'hystérésis vis-à-vis des tensions antérieurement appliquées.

3.2 Courants thermostimulés. - Pour les mesures de courants thermostimulés $i_{\text {TS }}$, nous avons opéré de la façon suivante : l'échantillon est courtcircuité à l'ambiante puis refroidi jusqu'à $130 \mathrm{~K}$. A cette température, on applique $V$ et on effectue une remontée linéaire de température jusqu'à l'ambiante à la vitesse de $5 \times 10^{-2} \mathrm{~K} \mathrm{~s}^{-1}$ au cours de laquelle on mesure $i_{\mathrm{TS}}(T)$. On stabilise alors la température à l'ambiante pendant $5 \mathrm{~min}$ environ puis on refroidit lentement l'échantillon en mesurant le courant de conduction $i_{\mathrm{c}}(T)$. Les courbes de la figure 5 sont relatives à un échantillon ayant un comportement de type 2 .

Le cycle 1 correspond à une tension appliquée de $1 \mathrm{~V}$. La partie basse température de sa courbe $i_{\text {Ts }}$ 


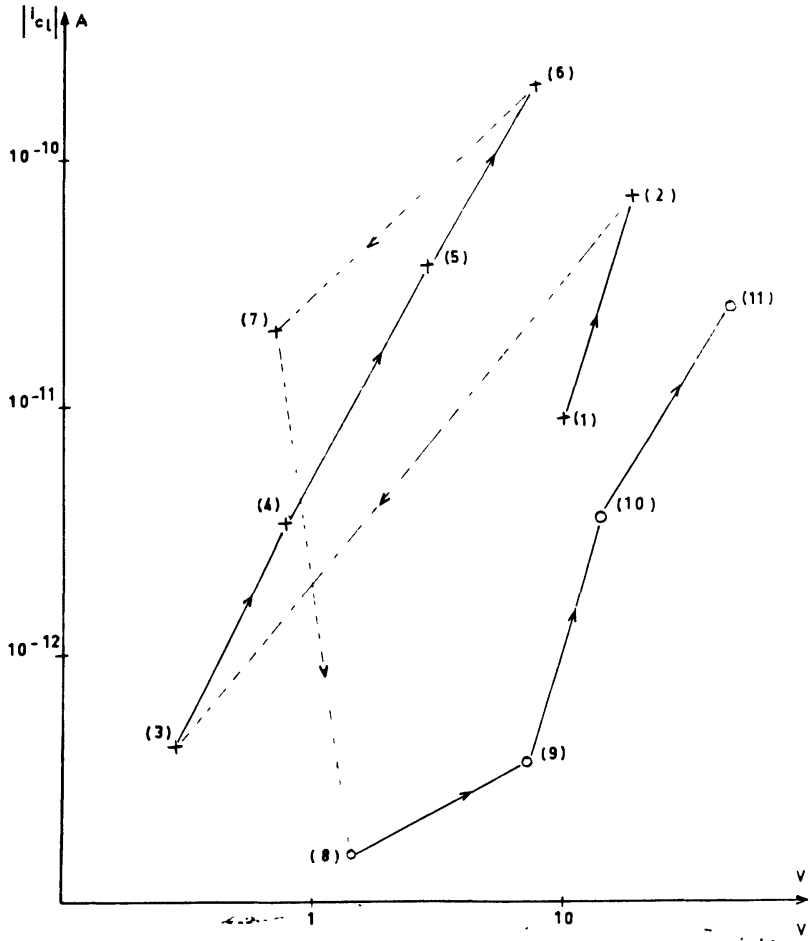

Fig. 4. - Courants de conduction à $130 \mathrm{~K}$ pour différentes séquences de tensions appliquées. Les nombres affectés à chaque point indiquent la chronologie des mesures. La séquence 8-9-10-11 correspond à des tensions inverses.

[Conduction currents at $130 \mathrm{~K}$ for different applied voltage sequences. The number indexing each point is referred to the chronology of measurements. The 8-9-10-11 sequence corresponds to reversed biases.]

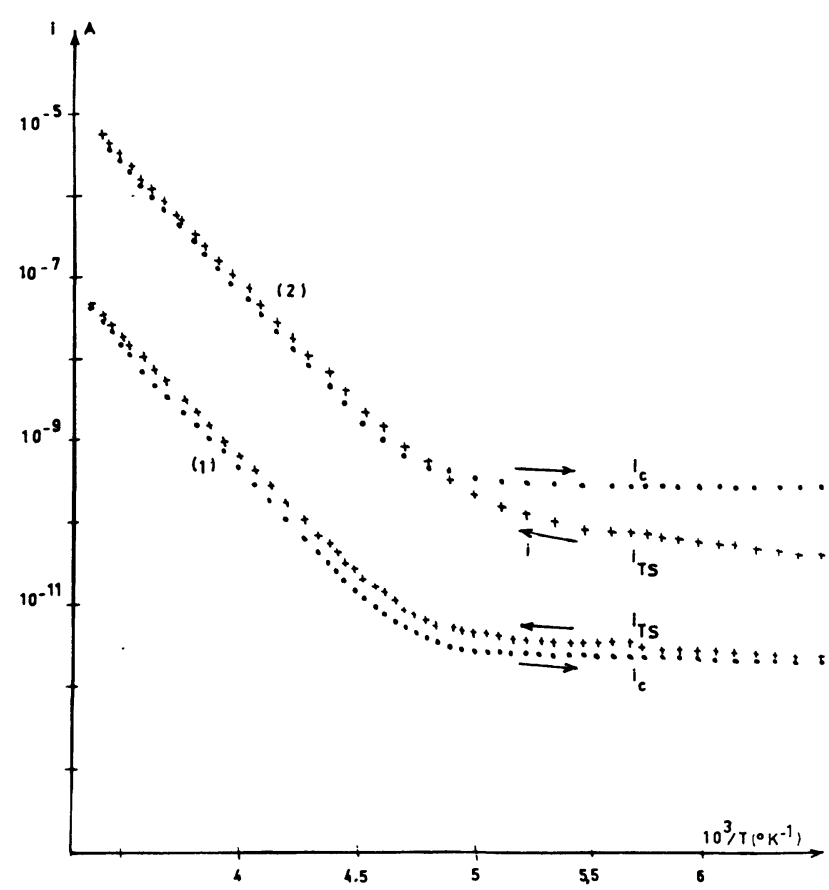

Fig. 5. - Cycles courants thermostimulés-courants de conduction. (1) : $V=1 \mathrm{~V} ;(2): V=100 \mathrm{~V}$.

[Thermostimulated currents-conduction currents cycles. (1) $V=1 \mathrm{~V} ;(2): V=100 \mathrm{~V}$.] présente un pic de thermocourant faiblement visible, superposé au palier du courant de conduction. Ce pic peut être dû à une libération de porteurs piégés. A plus haute température, on retrouve le courant de la conduction activée. Comme il est normal, la courbe de conduction subséquente se situe légèrement au-dessous de la courbe $i_{\text {TS }}$.

A des tensions élevées, par exemple pour le cycle 2 , la situation est différente puisque la partie basse température du palier de $i_{\mathrm{c}}$ se situe au-dessus de la courbe $i_{\mathrm{TS}}$. Ceci traduit une évolution du courant $i_{\mathrm{cl}}$, correspondant à $R_{1}$, entre le début et la fin du cycle. Le renforcement de $i_{\mathrm{cl}}$ semble donc induit par la circulation d'un courant activé important, résultant de l'application d'une tension élevée jusqu'à la température ambiante. L'étude de l'influence de $V$ sur les paliers apparait complémentaire de ces derniers résultats, ce qui établit le caractère semi-permanent du phénomène.

3.3 Mise EN ÉVIDENCE DES CHARGES D'ESPACE. Parmi les causes susceptibles d'induire une modification semi-permanente des propriétés électriques des échantillons, nous avons retenu l'hypothèse de charges d'espace piégées et tenté de vérifier leur existence.

3.3.1 Mesures de distributions de potentiel à la température ambiante. - En l'absence de champ appliqué, les mesures de distribution de potentiel montrent que le volume intérieur de l'échantillon court-circuité est à un potentiel supérieur de 0,1 à $0,2 \mathrm{~V}$ à celui des électrodes. Cette observation est en accord avec le relèvement superficiel des bandes qu'indique Adler [15] pour des films de $\mathrm{Te}_{39} \mathrm{As}_{36} \mathrm{Si}_{17} \mathrm{Ge}_{7} \mathrm{P}_{1}$ en contact avec des électrodes de molybdène et de graphite.

Lorsque des tensions de 10 à $50 \mathrm{~V}$ sont appliquées à l'échantillon, les courbes de répartition de potentiel permettent de montrer qu'une charge d'espace négative se développe au voisinage de l'anode et une charge d'espace positive plus réduite au voisinage de la cathode. L'étude systématique de ces hétérocharges n'a pas été réalisée car seule la preuve de leur existence et leur signe étaient recherchés.

3.3.2 Courants transitoires de charge et de décharge à la température ambiante. - Lorsqu'on applique, à la température ambiante, une tension constante à l'échantillon, on constate dans tous les cas, que le courant $i_{\mathrm{ch}}$ croît vers sa valeur $i_{\mathrm{c}}\left(T_{0}\right)$ du régime permanent ; après suppression de la tension et la mise en court-circuit de l'échantillon, on observe un courant transitoire de décharge $i_{\mathrm{d}}$ décroissant.

La présence du courant de décharge révèle qu'il y a eu précédemment séparation des charges sous l'action du champ électrique. Cependant, la constitution de telles hétérocharges se traduit généralement par une décroissance du courant de charge $i_{\mathrm{ch}}$ vers une valeur permanente [16]. La croissance observée 
nous oriente vers un processus de formation moins simple.

Par ailleurs, la distinction entre les deux types de comportements définis au paragraphe 3.1 .1 peut être poursuivie dans ces mesures. Ainsi le cas 1 est caractérisé par une constante de temps $\tau_{0}$ du transitoire de charge $i_{\text {ch }}$ de l'ordre de $15 \mathrm{~min}$; par une valeur relativement réduite du courant permanent; et par un courant $i_{\mathrm{d}}$ important et décroissant lentement de telle sorte que la charge mesurée peut atteindre quelques $10^{-7} \mathrm{C}$. Dans le cas 2 , la constante de temps $\tau_{0}$ est plus petite $(<2 \mathrm{~min})$, le courant permanent $i_{\mathrm{c}}$ est plus grand, $i_{\mathrm{d}}$ est plus faible et décroît rapidement, et la charge mesurée ne dépasse pas $10^{-10} \mathrm{C}$.

3.3.3 Thermocourants de dépolarisation. - Les mesures de courants de conduction et de courants thermostimulés se terminant à $130 \mathrm{~K}$, on courtcircuite à cette température l'échantillon. Puis, après avoir attendu le temps nécessaire pour que le courant soit stabilisé, on effectue une remontée linéaire de température à la vitesse de $5 \times 10^{-2} \mathrm{~K} \mathrm{~s}^{-1}$ au cours de laquelle on mesure le courant de dépolarisation thermostimulé $i_{\mathrm{dST}}$. Les courbes ainsi obtenues (Fig. 6), comportent, dans tous les cas, deux pics complexes $P_{1}$ et $P_{2}$ dont les sommets se situent respectivement vers $180-200 \mathrm{~K}$ et $300-310 \mathrm{~K}$. Le comportement de type 1 est caractérisé ici par l'existence d'un transi-

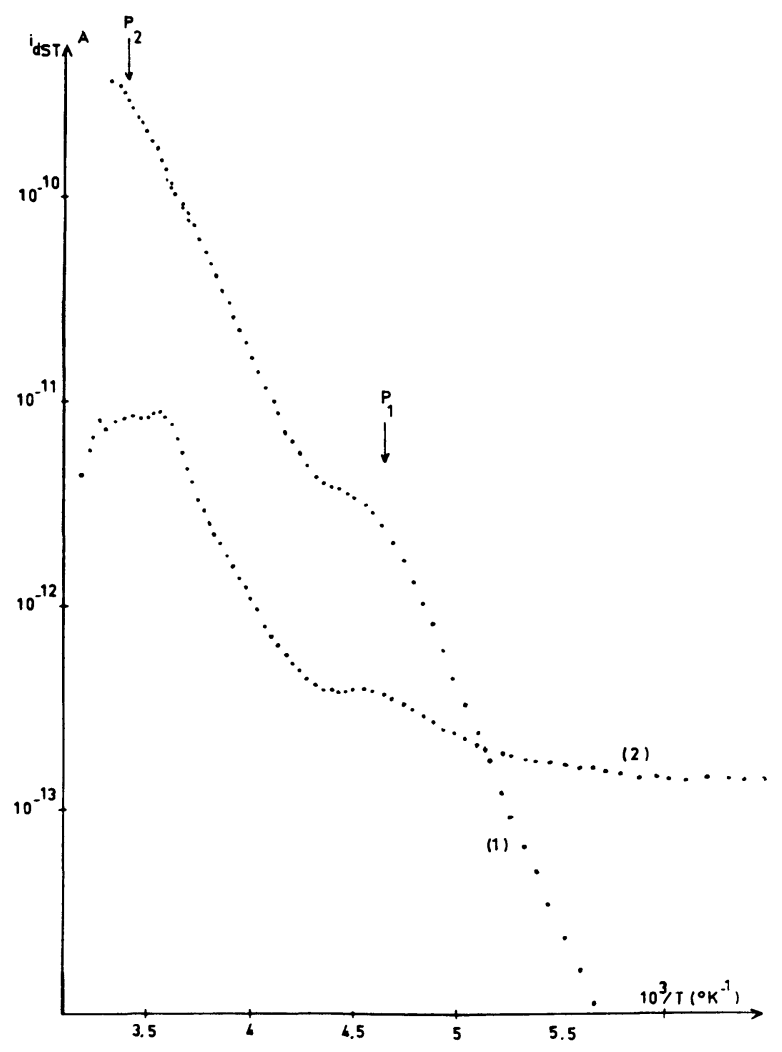

Fig. 6. - Courants de thermodépolarisation correspondant aux courbes de conduction (1) et (2) de la figure 1.

[Thermodepolarization currents corresponding to the conduction curves (1) and (2) of figure 1.] toire bref après la mise en court-circuit à $130 \mathrm{~K}$. Il en résulte que la croissance du pic $P_{1}$ est exponentielle, l'énergie d'activation étant comprise entre 0,47 et $0,54 \mathrm{eV}$ (courbe 1). Dans le deuxième cas (courbe 2), le courant après la mise en court-circuit ne décroît que lentement; le thermocourant subséquent ne varie que très peu dans toute la gamme de températures inférieures à celle du sommet de $P_{1}$ ce qui rend impossible la détermination d'une énergie d'activation. Par ailleurs, l'intensité du pic $P_{2}$ est plus faible que dans le premier cas.

3.3.4 Stabilité des échantillons. - Ce problème doit être posé dans le cadre plus général de la stabilité des amorphes. On sait en effet que ces solides se trouvent dans un état métastable et, de ce fait, une évolution lente et spontanée des propriétés est pratiquement toujours observée sur de très longues durées. Cette évolution peut devenir rapide lorsqu'on effectue des traitements thermiques à température élevée, ou électriques sous champs intenses.

Dans notre cas, nous avons constaté que les échantillons d'un lot, issus d'une même carotte, pouvaient présenter d'emblée un comportement de type 1 ou de type 2. De plus, certains d'entre eux primitivement de type 2, peuvent, après plusieurs mois, acquérir un comportement de type 1. Il semble impossible d'attribuer cette évolution à un recuit, car la température maximum atteinte est de $300 \mathrm{~K}$, ou à l'action du champ électrique, celui-ci ayant été maintenu à des valeurs faibles $\left(1 \mathrm{kV} \mathrm{cm}{ }^{-1}\right.$ au maximum dans les cas considérés). Cependant, les mesures effectuées donnent des résultats reproductibles lorsque la durée de l'ensemble des mesures n'est pas trop étendue.

4. Interprétation. - 4.1 DisCUSSION DES RÉSULTATS EXPÉRIMENTAUX. - Certains de nos résultats traduisent le comportement habituel des chalcogénures et peuvent être interprétés à l'aide des modèles exposés au paragraphe 1 .

- Le courant à l'ambiante et dans toute la région de conduction activée est dû à des porteurs libres de type $p$. L'énergie d'activation, légèrement inférieure à la moitié du gap optique, indique que le niveau de Fermi est sensiblement au milieu de la bande interdite.

- Le courant peu activé apparaissant à basse température, peut être attribué à une conduction électronique par sauts entre les états localisés du centre de la bande interdite. Cette hypothèse, conforme aux connaissances actuelles sur les chalcogénures, semble appropriée à nos mesures bien que celles-ci n'apportent pas la preuve que la loi de Mott leur soit applicable.

Par contre, l'évolution de la hauteur des paliers ne peut recevoir d'explication satisfaisante dans le cadre des théories habituelles. Nous ne pouvons pas, en effet, admettre que cette évolution soit due au recuit comme dans les expériences de Fagen et 
Fritzsche [5]. Cependant, nos résultats ont mis en évidence l'influence primordiale des tensions antérieurement appliquées. Ceci nous conduit à proposer un modèle qui, contrairement aux modèles existants, tient compte de la présence, dans l'état OFF, de charges d'espace. Ce modèle doit permettre d'expliquer la formation d'hétérocharges bien que le contact soit injectant pour les trous. Il doit caussi rendre compte des courants transitoires croissants alors que généralement, lorsque se forme une hétérocharge, ils sont décroissants [16].

4.2 DESCRIPTION DU MODÈLE PROPOSÉ. - On suppose, comme le font Godson et Hirsch [17] que les régions superficielles des échantillons sont plus résistantes que le volume. Il est naturel en effet d'admettre que la densité de pièges est plus grande en surface où le désordre est plus grand ce qui provoque une diminution de la mobilité effective des trous en présence de pièges peu profonds conformément à la relation [18], [15]

$$
\mu_{\mathrm{D}}=\mu_{0} \frac{p_{0}}{p_{0}+p_{\mathrm{t}}}
$$

où $\mu_{0}$ est la mobilité en l'absence de pièges, $p_{0}$ et $p_{\mathrm{t}}$ sont les densités des trous dans la bande et dans les pièges.

L'échantillon serait alors constitué de 3 régions (Fig. 7) : $a$ et $c$ étant les couches superficielles et $b$ le volume.

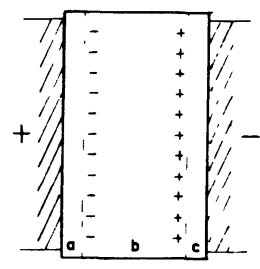

Fig. 7. - Schéma du modèle à trois couches.

[Three layers model.]

Lorsqu'on applique une tension, les trous sont injectés à partir de l'anode mais, leur mobilité dans la région $b$ étant plus élevée que dans la région superficielle $a$, celle-ci ne peut fournir assez de trous au volume et y remplacer ceux qui sont drainés par le champ. Selon Adler, les centres $C_{3}^{+}$peuvent libérer des trous.

$$
C_{3}^{+} \rightarrow C_{3}^{0}+e^{+}
$$

et il apparaît, dans le volume du côté de l'anode une charge négative due à un excès de centres $C_{1}^{-}$. Parallèlement, il se produit, à la limite de la région $c$, une accumulation de trous qui sont piégés par les centres $C_{1}^{-}$:

$$
C_{1}^{-}+e^{+} \rightarrow C_{3}^{0} \text {. }
$$

La formation de ces hétérocharges induit une augmentation du champ à l'intérieur des couches $a$ et $c$ [19] et, si les couches sont assez fines, il peut y avoir transfert d'électrons, par effet tunnel, à travers la barrière cathodique. Le courant correspondant se superpose alors au courant de trous et il apparaît à basse température, où il donne des paliers. Nous supposons qu'une partie des trous injectés vient remplir les pièges de la couche $a, \mathrm{y}$ réduisant le nombre de pièges vides et augmentant la mobilité réelle des trous libres dans cette région [15].

Après suppression de la tension, les charges d'espace sont progressivement libérées des pièges ; selon Adler, la charge d'espace en regard de la cathode serait plus stable car elle correspond à une modification structurale $\left(C_{1}^{-} \rightarrow C_{3}^{0}\right)$.

\subsection{APPliCATION AUX RÉSUltats EXPÉRIMENTAUX}

- Si on admet que la différence de comportement des types 1 et 2 est due à l'épaisseur de leurs couches superficielles respectives, le modèle précédent permet d'expliquer la plupart de nos résultats expérimentaux :

Le comportement de type 1 serait associé à des couches épaisses. Dans ce cas, en effet, le nombre d'électrons traversant la barrière cathodique n'est jamais suffisant pour donner un courant électronique observable dans nos conditions expérimentales. Le courant de trous à température ambiante n'est pas commandé par le champ dans le volume $b$ de l'échantillon car cela donnerait un courant de charge décroissant, mais par la résistance des couches superficielles ; le piégeage progressif des trous injectés dans la couche $a$ augmentant la conductivité de celle-ci, on observe un transitoire de charge croissant. Le courant de décharge est dû à la neutralisation partielle, par déplacement des porteurs à travers le volume, des hétérocharges formées sous tension.

Dans le cas 2, les couches superficielles sont minces. En présence de la charge d'espace, le champ au voisinage de la cathode peut devenir assez important pour qu'il y ait injection directe d'électrons à travers la barrière. Les paliers qui en résultent ont une intensité qui crôit donc avec l'importance de la charge d'espace piégée. Ainsi s'explique que le passage d'un courant de trous important, sous l'action d'une tension élevée à $300 \mathrm{~K}$, produise une augmentation du courant d'électrons (Fig. 5). Par ailleurs, la persistance d'une fraction de cette charge d'espace rend compte de l'hystérésis qui apparaît sur la figure 4 . En ce qui concerne le courant à température ambiante, le transitoire de charge est bref et le courant permanent plus élevé que dans le cas 1 car la zone de piégeage des trous injectés occupe très vite toute la couche $a$. A la dépolarisation, les charges d'espace disparaissent principalement par échange direct avec les électrodes [20] ; la charge recueillie dans le circuit de mesure à l'ambiante est alors beaucoup plus faible et l'intensité du pic $P_{2}$ réduite. 
5. Conclusion. - La mesure des courants dans nos échantillons a mis en évidence des comportements inhabituels pour les chalcogénures. L'effet d'hystérésis vis-à-vis des tensions préalablement appliquées a pu être attribué à la formation de charges d'espace de type hétérocharge dont on a vérifié l'existence. Le modèle proposé a rendu possible une interpréta- tion cohérente des résultats. En particulier, l'hypothèse simple d'une variation d'épaisseur des couches superficielles, a permis de distinguer les cas 1 et 2 . Ce travail sera poursuivi dans le but de confirmer le modèle, notamment par l'étude de la région superficielle et par des mesures de constantes diélectriques aux très basses fréquences.

\section{Bibliographie}

[1] Street, R. A., Mott, N. F., Phys. Rev. Lett. 35 (1975) 1293.

[2] Anderson, P. W., Phys. Rev. Lett. 34 (1975) 953.

[3] Adler, D., Yoffa, E. J., Phys. Rev. Lett. 36 (1976) 1197.

[4] Adler, D., Arntz, F. O., Flora, L. P., Mathur, B. P., ReInhard, D. K., 5th Int. Conf. on Amorphous and Liq. S.C., Taylor and Francis (1973) 859.

[5] Fagen, E. A., Fritzsche, H., J. Non Cryst. Solids 2 (1970) 170.

[6] Vezzoli, G. C., Walsh, P. J., Doremus, L. W., J. Non Cryst. Solids 18 (1975) 333.

[7] Motт, N. F., Philos. Mag. 19 (1969) 835.

[8] Pike, G. E., Seager, C. H., 5th Int. Conf. on Amorphous and Liq. S.C. Taylor and Francis (1973) 147

[9] Henisch, H. K., Fagen, E. A., Ovshinsky, S. R., J. Non Cryst. Solids 4 (1970) 538.

[10] Van Roosbroeck, W., J. Non Cryst. Solids 12 (1973) 232.

[11] Mackovski, J. M., Samueli, J. J., Kumurdjian, P., J. Non Cryst. Solids 15 (1974) 279.

Mackovski, J. M., Samueli, J. J., Kumurdjian, P., BourDarios, G., J. Non Cryst. Solids 8-10 (1972) 985.
[12] Madru, R., Maitrot, M., C.R. Hebd. Séan. Acad. Sci. Paris 270 (1970) 377

[13] Fornazéro, J., Siblini, A., Communication Privée (1978).

[14] Fagen, E. A., Fritzsche, H., J. Non Cryst. Solids 2 (1970) 180.

[15] AdLer, D., 7th Int. Conf. on Amorphous and Liq. S.C., Stevenson (1977) 695.

[16] Mathews, N. F. J., Warter, P. J., Phys. Rev. 144 (1966) 610.

[17] Godson, S. M., HIRSCH, J., 7th Int. Conf. on Amorphous and Liq. S.C. Stevenson (1977) 770.

[18] Motт, N. F., Davis, E. A., Electronic Processes in Noncrystalline Materials Oxford (1971) p. 209.

[19] Freeman, J. R., Kallman, H. P., Silver, M., Rev. Mod. Phys. 33 (1961) 553.

[20] VAN TuRnhout, J., Thermally Stimulated Discharge of Polymer Electrets Elsevier (1975) 15. 